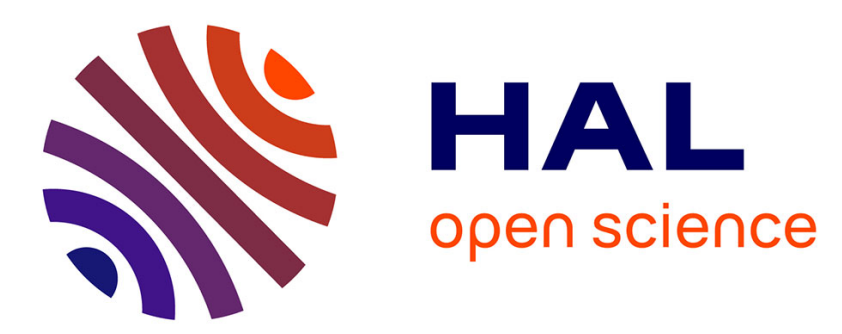

\title{
Comment penser la médiation inscrite dans les outils et leurs dispositifs: Une approche par le système artefactuel
}

Serge Agostinelli

\section{- To cite this version:}

Serge Agostinelli. Comment penser la médiation inscrite dans les outils et leurs dispositifs: Une approche par le système artefactuel. Distances et savoirs, 2009, 7, pp.355-376. hal-00487211

\section{HAL Id: hal-00487211 \\ https://hal.science/hal-00487211}

Submitted on 28 May 2010

HAL is a multi-disciplinary open access archive for the deposit and dissemination of scientific research documents, whether they are published or not. The documents may come from teaching and research institutions in France or abroad, or from public or private research centers.
L'archive ouverte pluridisciplinaire HAL, est destinée au dépôt et à la diffusion de documents scientifiques de niveau recherche, publiés ou non, émanant des établissements d'enseignement et de recherche français ou étrangers, des laboratoires publics ou privés. 


\section{Comment penser la médiation inscrite dans les outils et leurs dispositifs}

\section{Une approche par le système artefactuel}

\section{Serge Agostinelli}

LSIS UMR CNRS 6168, Université Paul Cézanne

Faculté des Sciences et Techniques de Saint-Jérôme

Avenue Escadrille Normandie-Niemen

F-13397 Marseille cedex 20

serge.agostinelli@univ-cezanne.fr

RÉSUMÉ. Les outils sont des moyens techniques et cognitifs mais aussi un processus social et culturel révélateur d'une action située et de connaissances partagées et distribuées. Les outils et dispositifs sont des constructions humaines et leur développement dépend étroitement du mode opératoire spécifique et de la réflexion des utilisateurs sur les finalités. Le processus artefactuel permet de modéliser ces aspects.

ABSTRACT. Tools are technical and cognitive resources, but also a social process and cultural indicative of situated action or shared and distributed knowledge. The tools and devices are human constructions and their development depends on the specific procedure, think of the users on the ends. The artifactual process allows a model of these aspects.

MOTS-CLÉS : artefact, processus, culture, affordance, anthropologie, communication.

KEYWORDS: artifact, process, culture, affordance, anthropology, communication.

DOI:10.3166/DS.7.355-376 @ Cned/Lavoisier 


\section{Introduction}

Cet article a pour but de proposer une posture épistémologique pour aider à comprendre les relations hommes-outils-culture. Cette posture propose un modèle artefactuel de ces relations qui pourrait permettre l'analyse des situations de médiation du savoir. Elle autorise la mise en synergie des modèles psychologiques de construction des connaissances avec les pratiques pédagogiques sous-tendues par les modèles didactiques et communicationnels. Nous cherchons particulièrement à clarifier avec quels concepts et méthodologies, nous pouvons penser la médiation inscrite dans ces outils et ces dispositifs. La médiation étant envisagée comme l'interaction amplifiée par le média (ici, les outils et dispositifs). En d'autres termes, nous pensons que l'organisation cognitive et l'accès à information dépendent des interactions, dans des situations instrumentées d'individus et d'outils qui sont des inscriptions du monde. Ainsi, nous nous inscrivons dans le courant de « l'action située » issue de l'approche vygotskienne que l'on retrouve chez Engeström (2000) et montre l'importance du rôle du contexte technique et culturel associé aux approches instrumentales et dynamiques de l'activité chez Rabardel (1995).

Dans cette perspective, la posture épistémologique est née de l'exigence d'envisager les situations de communication instrumentée comme un phénomène complexe plus large que les champs disciplinaires qui la questionnent. Cette vision anthropologique de la communication considère les outils comme les constructions d'une culture commune utilisées avec des règles établies dans un contrat situé. Ce sont ces règles implicites qui organisent une communication socialement élaborée et collectivement validée. Notre posture rejette donc les approches disciplinaires qui réduisent les outils à des ressources pour l'action. De fait, nous envisageons les outils comme un système qui met en relation un utilisateur, un outil, une connaissance, une situation et un contexte. C'est ce que nous appelons un "système artefactuel » (Agostinelli, 2003). Un tel système est avant tout, un système relationnel et représentatif pour penser le monde. Il se construit dans la relation (cf. Norman, 1993) et autorise la compréhension d'une situation de communication dans un contexte.

De nombreux travaux (Vygotsky, 1978 ; Suchman, 1987 ; Rabardel, 1995 ; Brassac, 2003 ; Folcher et Rabardel, 2004 ; Relieu et al., 2004 ; Hutchins, 2005...) ont montré qu'il n'était pas possible de comprendre l'activité humaine en dissociant l'homme des outils. Tous ont montré l'importance du contexte, des situations, des conditions particulières de médiation constituées par les individus, les connaissances, les artefacts. Ces travaux envisagent le rôle des artefacts dans ce qu'ils ont de culturel au sein d'une communauté où les outils sont le produit d'une activité humaine et auxquels, les membres attribuent des intentionnalités culturelles. Ils se donnent en fait pour objectif essentiel d'appréhender l'organisation du monde et les activités productrices comme des performances de la culture dans laquelle les connaissances sont considérées comme des œuvres humaines faisant l'objet d'une 
transmission culturelle socialement organisée (Meyerson, 1995). Les œuvres sont ici sélectivement organisés et utilisés pour leur valeur communicative (Hymes, 1967).

Dès lors, penser la médiation inscrite dans ces outils revient à se demander :

- Quel rôle jouent les outils dans la genèse des connaissances d'un individu à l'occasion des transformations de l'environnement?

- Comment la double fonction communicative et représentative des outils et/ou des dispositifs peut-elle autoriser la construction de sens et l'appropriation des règles?

\section{Position du problème}

\section{Le constat}

Aujourd'hui, la réalité de l'édition numérique, qu'elle soit à visées éducatives ou non, nous conduit à dépasser les approches trop étroites qui délimitent les analyses de l'organisation humaine et de l'accès aux savoirs associés, aux points de vue exclusifs de l'individu, des réseaux sociaux, des apprentissages ou de l'émergence de nouvelles pratiques. Nous entendons par " édition numérique ", à la fois les informations issues de la numérisation de documents conçus pour d'autres supports (livre, film, ressources pédagogiques...) et les process de réalisation et de diffusion de ces informations. C'est-à-dire, la structuration de l'information qui permet de décliner simultanément et automatiquement des ressources de toutes natures en ressources numériques. Cette perspective structuraliste limite l'édition numérique à une approche réductionniste de l'information, c'est la structure qui influence et détermine la nature de l'information. Dès lors, la modélisation de l'organisation humaine dans ce domaine relève souvent d'une formalisation des documents, de leurs flux, de leurs traitements, et du niveau de responsabilité des acteurs associé à la dynamique des échanges (interruptions, parallélisme). Pour assurer la dynamique, les dispositifs disposent de fonctionnalités de navigation et d'interrogation. Dans l'exemple sur une plateforme éducative, un enseignant (qui dépose de la documentation) et un étudiant (qui consulte) sont définis par leur identifiant, leur mot de passe et leur email, leur groupe d'appartenance etc. Ce type de métamodèle structurant est complété par des aspects informatiques qui permettent sa mise en mettre en œuvre. En revanche, nous considérons que la réflexion sur les accès à l'information et aux connaissances est indissociable d'une compréhension de l'articulation qui existe entre le processus de transmission culturelle socialement organisée d'appropriation des connaissances et le rôle des outils. C'est-à-dire, la dimension située et partagée de la cognition inscrite dans un ensemble d'artefacts. De fait, la complexité des « choses » à observer en regard de la compréhension insuffisante et du manque de culture numérique dont nous faisons preuve, conduit à cette déduction surprenante que ces phénomènes observés échapperaient à une formalisation théorique. Dès lors, une forme d'empirisme s'installe sur les 
réflexions et induit un discours scientifique qui accorde une importance de premier plan aux faits ou " aux faires ». La culture numérique qui devrait passer par la reconnaissance d'une architecture structurelle (les outils) et d'une organisation fonctionnelle (les humains), dans le respect des règles collectivement construites et socialement validées (les usages), se limite alors, pour un individu, à mettre en œuvre des savoir-faire définis dans un domaine spécifique. Ces savoir-faire sont regroupés en types de tâches qui caractérisent un découpage des activités cognitives et manuelles sous-tendues par la maîtrise des outils.

Ces analyses prennent souvent une place importante dans les travaux scientifiques sur le domaine et dès lors, les réflexions sur les outils sont plus centrées sur ces pratiques (Bertrand, 2004 ; Campillo, 2008) ou la logique des usages (Perriault, 1989 ; Chaptal, 2007) que sur les modèles théoriques (Moersch, 2001 ; Robert, 2009). Bien sûr, même si ces analyses des pratiques numériques ont pour but de dégager les caractéristiques fondamentales (fonctions sociales, situations et problèmes correspondants, concepts fondamentaux, stratégies spécifiques...) et définir ainsi des invariants d'une culture numérique, les recherches sur les pratiques se constituent en connaissances transversales fondées sur des travaux de terrain et reconnues par l'interdisciplinarité issue des divers champs de publication. Elles se constituent comme acteurs d'un paradigme construit sur du vécu et leur implication dans la société de l'information.

\section{Le résultat}

$\mathrm{Au}$ regard de ce constat, deux options de réflexion sont le plus souvent retenues pour discuter les outils et leurs dispositifs. La première donne un sens au réel formel des outils pratiques, celui des technologies et les arguments sont « conformes » au sens intrinsèque du quotidien. La seconde amplifie le discours social sur un réel informel socialement construit, celui de la société de l'information et les arguments sont « recevables » par la société hic et nunc.

Quelle que soit l'option choisie, le discours sur l'édition numérique fonde les outils comme des arguments des nombreuses formes et normes de structuration et d'accès des informations. Cet amalgame entre les outils et l'activité humaine n'est pas spécifique à l'édition numérique. Par exemple, dans l'enseignement à distance, nous sommes dans le « learning » du e-learning et le « $\mathrm{e}$ » devient accessoire ; dans la e-démocratie, dans l'e-citoyen ou le e-commerce, le e-book, la e-édition... nous sommes toujours dans un discours « mou » qui amalgame les outils et les pratiques observées. Toutefois, les outils ne peuvent pas être limités à un discours, ils ont aussi une action sur le monde et pas seulement sur l'activité. Les outils sont plus que des vecteurs ou des supports d'informations qui n'ajouteraient ou ne retireraient rien au monde ni n'en changeraient l'épistémologie. D'une part, les outils sont une traduction fonctionnelle à nos besoins (Callon, 1986). Ils sont d'ailleurs, sans cesse, transformés par de nouveaux objets orientés par les choix nécessaires au caractère 
chaotique et fragile de l'élaboration d'une culture numérique. Cette traduction fonctionnelle autorise l'ensemble des discussions visant à aboutir à un consensus sur le discours social, grâce à quoi chaque nouvel outil se permet ou se fait attribuer l'autorité du savoir-faire et de l'agir au nom d'une pratique. Dès que le discours sur les outils amplifie le discours social, ils traduisent les activités humaines en une seule volonté dont ils deviennent le porte-parole. D'autre part, ils sont des ressources médiatrices pour l'action et autorisent la diversité des logiques d'usage d'un accès conjoint aux ressources pour l'action. C'est un processus de coconstruction qui passe par la production d'intelligibilité mutuelle et par la vérification des conditions de cette intelligibilité (Suchman, 1987). L'accent est mis ici sur l'importance des interactions qui reposent sur la construction d'une intelligibilité partagée. Cette intelligibilité autorise l'interprétation des éléments de la situation et l'accès aux ressources disponibles dans un environnement technologique, social et culturel particulier et plus ou moins stabilisé (Weill-Fassina et al., 1993). Enfin, les outils sont une forme temporelle de la représentation de l'homme et de son activité. C'est ce qui donne un sens au réel formel des outils et fait partie intégrante du processus d'acculturation numérique.

\section{La conséquence}

Il ne peut pas y avoir de réflexion sur les outils, si l'on ne prend en considération que les opportunités (ou les contraintes) d'ordre technique, économique, socioculturelles et les implications sociales qui en résultent. Comment envisager une culture numérique sans réfléchir aux outils par lesquels les objets de transmission des connaissances sont produits ou aux outils par lesquels les connaissances sont diffusées ? Le choix des outils crée des situations qui entraînent de nouvelles connaissances, qui modifient les outils. Les capacités cognitives s'adaptent ainsi aux outils et aux pratiques sociales dans lesquelles elles s'inscrivent (Gauvain, 2001). Les outils sont donc essentiels à tout type de mise en situation. Comment oublier l'importance théorique des outils dans la cognition ? Comment oublier que les outils relèvent aussi, d'une étude des technologies?

\section{Approche théorique du rôle des outils}

Suivant une interprétation vygotskienne des outils, les activités sont médiatisées par des outils et systèmes d'outils élaborés par les générations antérieures, c'est-àdire par la culture. Ces outils, en tant que médiateurs entre actions cognitives et activités situées, assument une double fonction : de transformation de l'activité ; de représentation (des objets, de leurs propriétés, et des transformations opérées).

Cette approche épistémologique exclut la position selon laquelle les outils ne sont que les moyens d'une pratique naturelle issue du « faire », et considère que l'appropriation des outils est l'essence même du développement de l'esprit. En 
d'autres termes les pratiques qui fondent les outils tirent leurs origines des objets, médiateurs de la culture et de sa transmission. L'individu s'en approprie les propriétés grâce aux rapports sociaux au cours desquelles des fonctionnements interindividuels médiatisés par des outils matériels peuvent se transformer en fonctionnements intra-individuels eux-mêmes médiatisés par des outils cognitifs.

Dès lors, si les connaissances et les outils cognitifs sont construits en contextes interactifs et issus des médiations socialement élaborées (Vygotsky, 1978) ; si les pratiques construites sont des réalités sociales collectivement négociées et distribuées (Bruner, 1991) ; si la réussite des interactions sur les processus de production d'intelligibilité mutuelle et d'accès partagé aux ressources de l'environnement repose sur une interprétation des éléments de la situation (Suchman, 1987) ; alors, l'importance des outils dans la construction des connaissances ne fait aucun doute et on ne peut plus se contenter d'une analyse des pratiques en termes de moyens. Ce qui nous contraint à étudier les outils dans leur complexité dynamique. On a donc absolument besoin d'un modèle qui envisage les outils comme un système cognitif incarné, c'est-à-dire capable de perception et d'action en même temps que d'intelligence. Ce système met en relation des individus, des outils, des connaissances, une situation et un contexte. C'est pourquoi nous proposerons un modèle qui permet cette mise en relation : il s'agit du modèle artefactuel détaillé plus loin.

Les outils ne sont pas seulement des objets techniques. Elles sont des moyens techniques, cognitifs et culturels. Utiliser un navigateur sur le web requiert autant d'habiletés techniques qu'une activité de représentation cognitive. L'outil est ainsi représenté dans l'esprit de l'internaute, il transforme alors les processus mentaux, les stratégies cognitives d'exploration, l'activité d'apprentissage. Les outils ne sont pas de simples accessoires à l'activité humaine, ils la transforment (Vygotsky, 1985 ; Tikhomirov, 1974), ils l'amplifient. Les outils sont à la fois le produit, d'une activité humaine, de pratiques sociales et les déclencheurs d'une activité intellectuelle. Ils sont aussi des «creusets » de la connaissance humaine socialement partagée à un moment donné (Norman, 1993 ; Lave, 1988).

L'intérêt des outils réside donc, dans les diverses formes d'organisation qu'elles peuvent proposer et d'actions qu'elles peuvent susciter pour l'accès à l'information scientifique éditorialisée. Les outils sont organisés comme une connaissance communément partagée pour servir de système commun informationnel. Ici, les outils ont trois fonctions. La première est organisante : ils organisent les connaissances situées. La deuxième est informationnelle : ils sont la source des médiations entre les connaissances et les pratiques. La troisième est régulatrice des deux premières. Notre point de vue se fonde ici, sur l'étude des outils en tant que phénomène social et culturel révélateur d'une action située et d'une connaissance partagée et distribuée. 


\section{Cadres théoriques}

Suivant une approche interactionnisme, l'acquisition des connaissances peut-être envisagée suivant deux approches de la relation individu-outil : la première va de l'individu vers le social ; la seconde à l'inverse par du social pour aller vers l'individu et ajoute une médiation. Ces deux options théoriques peuvent être retenues pour discuter de la mise en relation entre les connaissances et un individu.

\section{Une théorie prototypique des significations}

Cette option théorique induit une acquisition des connaissances fondée sur un processus cognitif de traitement des informations dans lequel les résultats du traitement génèrent des entrées pour d'autres traitements. Les connaissances sont stockées sous la forme de blocs qui constituent la base de connaissances disponibles. Ces blocs sont reliés par des réseaux sémantiques qui structurent les connaissances en schémas prototypiques utilisables dans plusieurs situations. Ces schémas sont instanciés par les informations de la situation particulière. C'est le modèle des significations à la fois prototypiques et procédurales. La théorie des prototypes (Rosch, 1975) est une théorie de la catégorisation. Elle est particulièrement opérante si les pratiques observées sont définies par des propriétés que toutes les situations partagent ; alors aucune situation d'une catégorie (en d'autres termes, aucun applicatif ou logiciel) ne devrait être une meilleure solution (applicatif) que d'autres. Or, si l'on observe les pratiques supportées par les outils, on s'aperçoit que les propriétés importantes pour la catégorisation sont moins des propriétés perceptives que des propriétés fonctionnelles (par exemple, tous les logiciels de mail chargent du courrier, ils ont tous la même fonctionnalité pourtant, aucun d'entre nous changerait le sien). Dans la catégorisation des outils intervient de manière centrale, le caractère finalisé des activités humaines.

Avec cette approche, les outils facilitent la navigation entre différentes situations (graphiques, calculatoires...) et permettent de tisser des liens entre les situations. Les individus accroissent ainsi leur réflexion sur les activités qu'ils sont en train de mener. Bien sûr, la question qui relève de la communication est ici de savoir quelles sont les relations (homme-outil-homme) que les individus sont susceptibles de construire, compte tenu des situations qu'ils rencontrent ou de l'état de la genèse de leurs connaissances. La difficulté techno-cognitivo-communicationnelle est bien dans le passage de la navigation entre les situations instrumentées proposées à la navigation entre les relations humaines sous-tendues. Comment présenter et lier les situations de communication en fonction du réseau social des relations? En fonction de l'activité (forum, chat...) et des pratiques (règles du social network), quels sont les liens sociaux et cognitifs que va tisser l'individu ? Si les situations instrumentées doivent refléter les relations conceptuelles, le «e » devient l'intermédiaire entre les champs conceptuels et les relations de l'homme : c'est la e-relation.

Cette vision, essentiellement liée au réel formel des outils et à leurs aspects structurants justifie la qualité les dispositifs. Les outils doivent devenir intuitifs pour 
parvenir à faire reconnaître leurs intentions. L'outil est cohérent à l'activité de façon à ce que l'individu l'utilise de manière adéquate, etc. Ainsi, la structure idéale de l'outil peut déterminer un degré de compétence des pratiques et on peut donc mesurer le degré d'efficience de l'utilisateur compétent. D'ailleurs, les pratiques constituent une structure pragmatique qui permet de décrire des aptitudes ad hoc développées par les utilisateurs dans des situations variées. Il sait mobiliser des ressources en situation, il a atteint la qualité optimale.

L'acquisition de cette qualité (habilité, expertise...) se fait grâce à une pratique durant laquelle l'individu dispose d'une certaine liberté au niveau du choix des outils. Le « $\mathrm{e}$ » assure particulièrement bien son rôle, en offrant suffisamment d'outils hightech, performants, puissants, aux individus expérimentés et suffisamment motivants, ludiques, faciles pour capter l'attention des plus prudents. Ce qui correspond aux canons technos sociaux actuels : la mondialisation des outils comme fondement du lien social qui accompagne une logique d'accélération et d'une systématisation de la vitesse. Nous sommes dans l'universalité du règne des outils et l'analyse ne parvient pas à dépasser les questions du dialogue homme machine qui renvoient à l'amalgame permanent entre l'instrumentation technique d'une action humaine et sa mise en œuvre effective par l'action des individus. Dès lors, lorsqu'on évacue le rôle des outils, comment savoir à quel niveau du développement des relations ou des opérations cognitives et sociales interviennent les activités basées sur les outils ?

\section{Une théorie de l'interaction}

Cette seconde option, se fonde sur la nature processuelle de la cognition, c'est un mouvement qui se développe de manière interactionnelle au cours de la vie (Goffman, 1973, 1974) c'est une adaptation au contexte. Ici, l'utilisateur compétent s'adapte aux buts qu'il cherche à atteindre. Il utilise les outils pour atteindre ses objectifs et peut même modifier son environnement de manière appropriée et effective. En fait, cette adaptation au contexte est autorisée par trois compétences fonctionnelles (compréhension d'une situation problème à résoudre, d'un cas soumis pour traitement) : L'utilisateur a un intérêt à utiliser cet outil (cet outil me permet de faire) ; il est conscient de sa pratique (voici ce que je peux faire avec); il reconnaît la situation dans laquelle cette pratique se produit (cet outil me change la vie). Bien sûr, la compétence étant entendue comme capacité réflexive. C'est-à-dire, tout ce que les individus connaissent (ou croient), de façon tacite ou discursive, sur les circonstances de leur action (et de celle des autres), et qu'ils utilisent dans la production et la reproduction de l'action.

Cette option envisage l'acquisition des connaissances comme un système de mise en relation des individus, des outils, des connaissances, une situation et un contexte. Les informations sont composées d'une collection de « données » séparées qui constituent un système périphérique (ou trophique) de traitement de l'information. Ce système est autonome par rapport au système global, qui recouvre les facultés « supérieures » de prise de décision, d'intentionnalité ou d'organisation. Le processus informationnel se transforme en processus décisionnels. Les processus 
décisionnels sont de nature cognitive et directement issus des rapports sociaux. Ils sont collectivement élaborés grâce aux outils servants à représenter les activités ; collectivement médiatisées par les outils et artefacts qui organisent les actions. C'est un modèle des médiations à la fois interactionnelles et contextuelles. Cette option induit aussi l'idée de pratiques sociales et la nécessité de penser l'individu d'abord par la communauté. Elle a l'avantage de mettre en lumière la dimension macro et globalisante des outils qu'elle présente comme une entité. Mais aussi, la dimension micro qui souligne les processus organisants animés par des comportements interconnectés (Weick, 1979) focalisés sur les interactions locales.

Ces deux points de vue (approche par les protptypique vs approche par les interactions), que l'on oppose le plus souvent, sont ici complémentaires et autorisent la compréhension à la fois des usages quotidiens et l'expression d'un monde en mouvement. Dès lors, l'organisation et l'accès à information numérique articulent les trois systèmes proposés par le schéma suivant.

Système global dans lequel, les connaissances vont se spécifier dans un domaine particulier. Les connaissances étant définies comme le savoir maîtrisé, reconnu, dans un domaine d'importance et qui

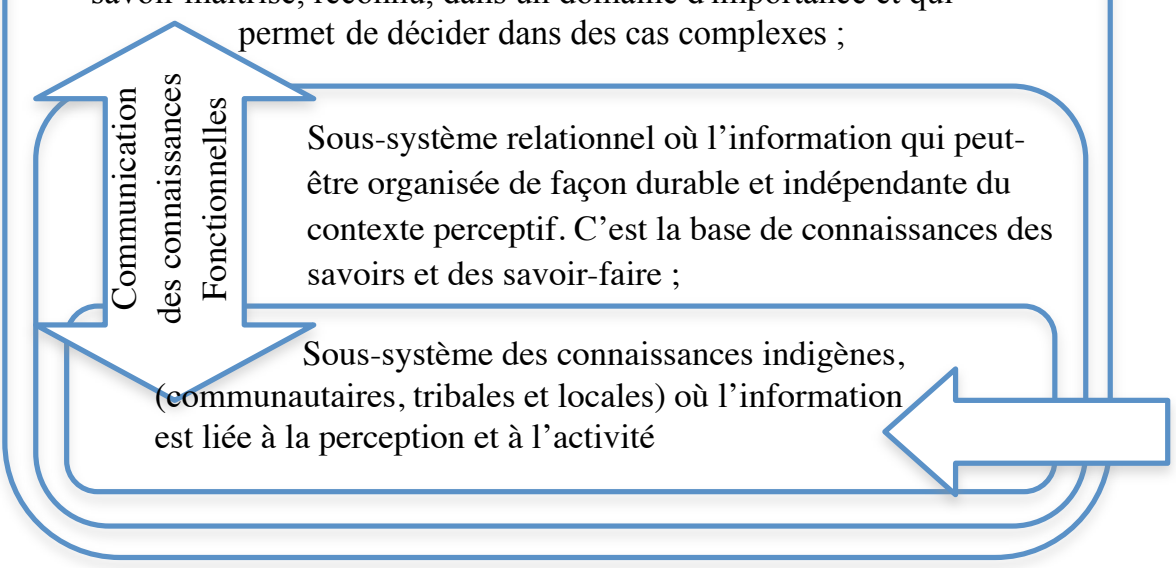

En d'autres termes, c'est un schéma de la médiation. Celui, dans lequel, l'acquisition de connaissances dépend de l'organisation de la base de connaissances et des relations entre l'organisation et les concepts qui entrent dans la situation. On fait ici l'hypothèse que l'individu dispose de savoirs et de savoir-faire. C'est le reflet du monde d'un individu et on définit alors cette partie de la pensée sur l'action en termes de base orientatrice. Elle est « un système ramifié de représentations de l'action et de son produit, des propriétés du matériel de départ et de ses transformations successives ainsi que toutes les indications dont se sert pratiquement le sujet pour exécuter l'action » (Galpérine, 1980, p. 169). 
L'individu a donc des représentations du monde que l'on peut qualifier d'initiales, elles ne sont pas des représentations scientifiques et sont probablement incomplètes, voire erronées ; on ne parle plus des manques, des lacunes, mais des transformations. L'acquisition de connaissance relève alors de la modification fonctionnelle que l'on a d'un objet matériel ou conceptuel. Toutefois, en faisant toujours l'hypothèse que les individus ont déjà leur vision du monde (même local), il convient d'accepter qu'ils aient aussi des connaissances antérieures qui font, non seulement partie de l'environnement d'apprentissage, mais qui font obstacle aux opérations de modification des représentations (Abric, 1997). C'est ce qu'on appelle l'obstacle épistémologique (Bachelard, 1996) qui se traduit par un conflit cognitif. Il peut être personnel lorsque les représentations de l'individu et de celles proposées par l'environnement ne correspondent pas. Il peut être social lorsque dans un groupe d'individu, les représentations individuelles s'entrechoquent. Dans les deux cas, on parle d'interaction, avec laquelle, la construction des connaissances joue, à chaque étape, un rôle central dans l'appréhension de l'environnement et sur d'éventuels effets de ces mêmes connaissances. Dès lors, les connaissances, qu'elles soient individuelles ou collectives, vont se spécifier dans un domaine particulier grâce à une communication des connaissances en situation, aux interactions, aux relations intersubjectives dans le partage d'une matérialisation de la connaissance (Lave, 1988). C'est ici, la volonté de mettre les connaissances dans l'accomplissement de l'action et de rendre compte de la construction et de la révision des connaissances individuelles contraintes par leurs interdépendances ou rendus possibles par cellesci. Dans ce modèle, la médiation a un rôle central, elle transforme le processus interpersonnel en processus intrapersonnel (Vygotsky, 1985).

Globalement, il s'agit de remettre au cœur des outils « ce qui avait été indûment enlevé pour l'attribuer à l'esprit ou au sujet » (de Formel et al., 1999, p. 29). Ainsi, on relance le rôle de l'environnement (technique) sur le social et l'individuel, par la prise en compte de l'implication de la culture (Bruner, 1991 ; Hutchins, 1995), de la médiation sociale (Vygotsky, 1985) et de la nature des situations (Goffman, 1973). De ce point de vue, « ce qui rend possible la pensée individuelle c'est l'existence d'un environnement stable de pensées, de conceptions, de représentations et de significations qui ne sont celles de personne, bref d'un " esprit objectif » dont les supports sont les pratiques, les us et coutumes, et les institutions d'une société » (de Formel et al., 1999, p. 28). Dans ce cas les outils appartiennent à un « système cognitif distribué » (Hutchins, 1995), ils deviennent des artefacts qui amplifient les aptitudes humaines, modifient la tâche et l'activité. Ces artefacts organisent notre vision du monde à travers les connaissances distribuées et les pratiques sociales partagées qu'ils autorisent (Bateson, 1972). Ils proposent des ressources pour la structuration et l'exécution d'actions humaines (Norman, 1993 ; Lave, 1988). Ils favorisent la réflexion, la pensée, et déclenchent une activité intellectuelle. Lorsqu'ils modifient les activités cognitives, ce sont les artefacts cognitifs (Norman, 1993). Lorsqu'ils modifient les activités de communication, ce sont « les artefacts communicationnels » (Agostinelli, 2003) qui amplifient la communication, organisent 
l'interaction humaine, modifient les modes de production de gestion et de traitement de l'information.

\section{La notion d'artefact}

Notre approche par les artefacts trouve sa source dans les objets envisagés comme des productions sociales de l'homme (Simondon, 1989) et dans la notion d'instrument, définit par les relations d'usage entre l'homme et l'objet (Rabardel, 1995). Pour Rabardel, l'outil médiateur de l'activité est reconsidéré en instrument et se définit à la fois par rapport à l'objet et au sujet de l'activité. L'instrument se compose donc de l'outil matériel ou symbolique, produit par le sujet ou d'autres et des modalités d'usage de cet outil par le sujet. La méditation peut alors exister entre le sujet et l'objet de l'activité (nous pensons qu'elle est identique à la médiation par l'outil de Engeström). Celle-ci peut être épistémique et autoriser la reconnaissance de l'objet à travers ses propriétés, son histoire, etc., ou pragmatique et concerner les actions de transformation sur l'objet. Elle trouve également ses racines dans la cognition distribuée et la théorie de l'activité qui se concentre sur la médiation par l'outil (Leontiev, 1984 ; Engeström, 1999). Les instruments techniques peuvent transformer les objets et l'environnement, les instruments psychologiques (que l'on appelle aujourd'hui l'artefact cognitif, Norman, 1993) réorganisent les structures cognitives du sujet.

L'artefact est un artis facta, un effet de l'art, une construction progressivement élaborée au cours de médiation pour atteindre la « quasi-réalité » de représentations destinées à être démantelées aussi méthodiquement qu'elles ont été imaginées afin de le rendre incontestable à l'utilisateur. Bien sûr, l'art est ici entendu comme une aptitude, une habileté à faire " quelque chose », mais aussi comme un ensemble de moyens, de procédés, de règles intéressant une activité, une profession. C'est aussi une activité conduite et considérée comme un ensemble de règles à observer ; un ensemble d'activités humaines créatrices d'ouvrages contenant les préceptes, les règles d'une discipline. L'effet ou influence est envisagé comme le résultat d'une action. Ce qui est produit par "quelque chose », qui provoque une action, une réaction. Bien sûr, tout art d'influence peut donner lieu à une subjectivité suivant le contexte et l'usage, les pratiques. Certaines œuvres d'art deviennent de «bons objets », de bonnes constructions car elles s'attachent au social tout en le fabriquant en partie avec la particularité d'adhérer par tous leurs points au corps social. D'autres, comme les objets techniques ou les objets fétiches (Latour, 1995), sont des objets détachés, objectifs, qu'on ne sait plus souvent lier au reste du monde social. Les effets de l'art, peuvent alors provoquer des erreurs d'interprétation, de compréhension, voire des réactions de rejet. Une vision contextuelle est donc nécessaire pour observer l'artefact. Il n'est ni de l'ordre des moyens, lesquels varient considérablement selon les points de vue, ni de l'ordre des fins car les possibles qu'il autorise ne sont pas à proprement parler, une fin en soi. Du coup, les pratiques ne sont plus l'essentialité de la relation homme-artefact comme solution unique au 
problème pris en compte (Rabardel, 1995) mais uniquement une solution immédiate, hic et nunc et non projective. En fait, les pratiques ne sont pas la manifestation d'une relation homme-artefact identique à elle-même qui apparaîtrait et réapparaîtrait à échéances variées, dans un univers qui la reconnaîtrait et qu'elle reconnaîtrait. Elles sont une concordance transitoire de divers composants, inférables par une lecture en contexte d'un modèle décisionnel qui met l'accent sur le rapport entre les actions observables et les décisions prises par les individus. De ce point de vue, la communication quotidienne en présentiel n'est pas plus « naturelle » qu'une communication instrumentée, les artefacts ne sont pas « hors nature ». Un artefact est d'abord une vue de l'esprit qui associe les interactions humaines, la médiation des outils, la cognition et les intentions de communiquer.

\section{Le processus artefactuel}

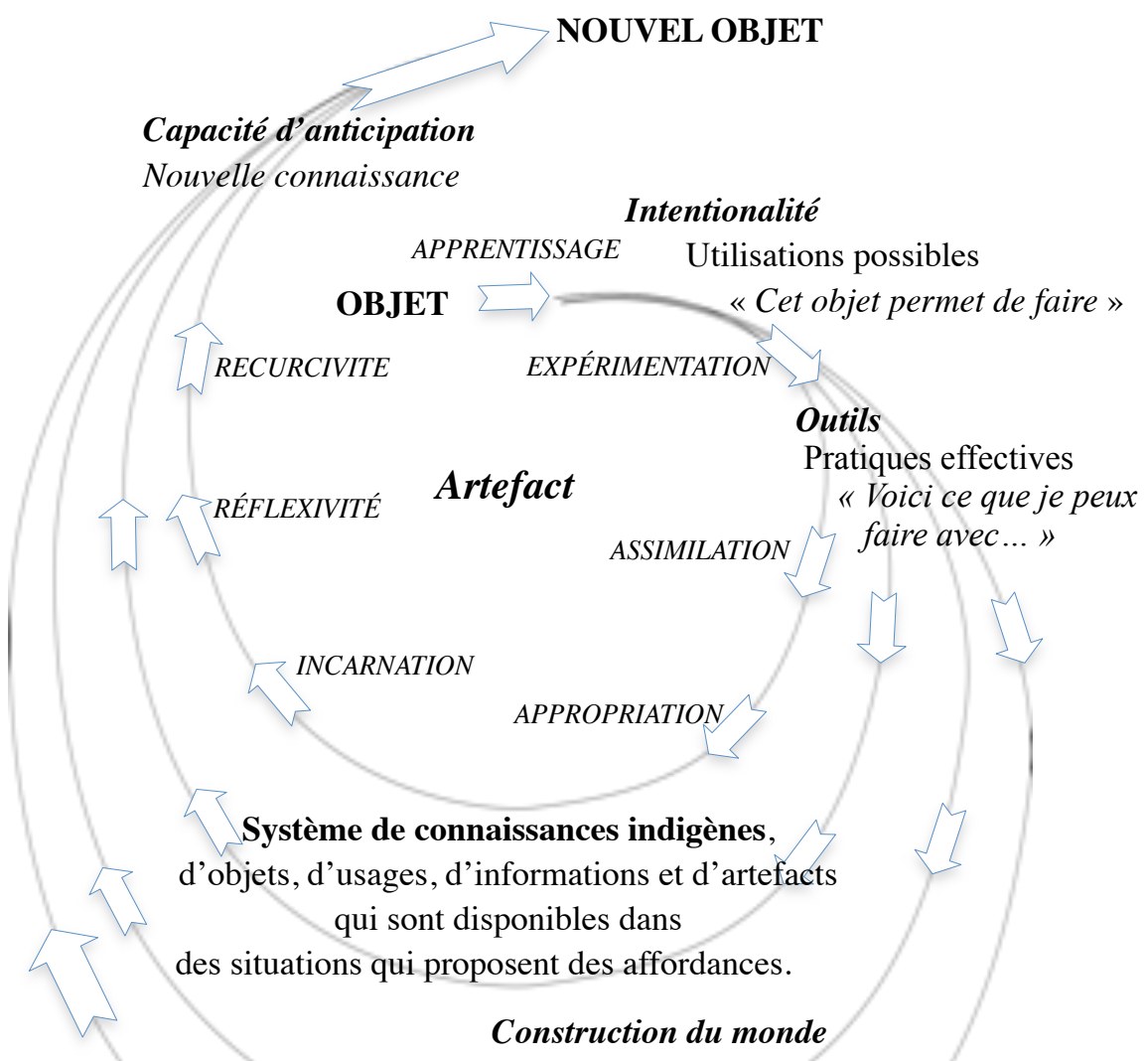

Médiation Vision personnelle et locale qui ne peut pas exister sans l'outil "Voici les règles d'usage de mon outil qui autorisent mon projet »

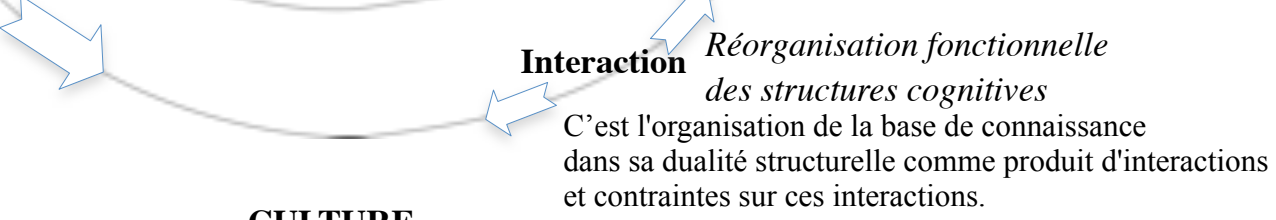

CULTURE et contraintes sur ces interactions. 
Avec le processus artefactuel, il s'agit de définir un cadre méthodologique pour appréhender l'analyse de la médiation inscrite dans les outils et leurs dispositifs. Nous regardons particulièrement leur propension à générer un espace commun de communication (Agostinelli, Metge, 2008) où l'environnement (artefact, culture, objets naturels, etc.) joue un rôle primordial dans la compréhension des situations. On insiste d'ailleurs sur la fonctionnalité des affordances (Gibson et al., 1997), par lesquelles un individu détecte les invariants structurels correspondant aux propriétés permanentes de cet environnement.

Le processus commence avec la découverte de l'objet (Rabardel, 1995) et les questions associées ; " à quoi ça sert ? en quoi est-ce fait ? etc. ». C'est l'intentionnalité donnée à l'objet (Andreucci et Ginestié, 2002) qui lui autorise une utilisation possible, " cet objet me permet de faire ». Avec l'apprentissage et les expérimentations, se construit une connaissance subjective liée à la relation personnelle que nous avons avec l'objet et avec cette connaissance, « voici ce que je fais avec... ». L'outil évolue ensuite vers un usage établi et considéré comme normal, il devient un outil personnel et annonce, par les termes « cet outil me change la vie... ", des actions sur, par, avec l'outil. C'est ici qu'intervient l'interaction qui relève de l'organisation et de la genèse des connaissances. La construction mentale des connaissances joue, à chaque étape, un rôle central dans l'appréhension de l'environnement, et dans d'éventuels effets sur ces mêmes connaissances. Dans la construction sociale (Berger et Luckmann, 1997), la réalité intersubjective fait l'objet de représentations conflictuelles en permanente (re)construction. Les représentations qui s'élaborent, traduisent les constructions intériorisées qui transforment le réel par étapes successives en réalité situationnelle. L'individu se construit alors par un système d'interactions. Ce système se traduit par la construction d'un contexte simultanément à l'avancement des échanges qui permettent la création ou l'amélioration d'un processus volontaire et réflexif. Ce processus autorise chez l'individu, une planification de son action pour l'atteinte du but grâce à une plus grande structuration de ce contexte et une meilleure gestion de son activité.

Dans la construction d'un monde personnel et local, la culture est « l'ensemble des descriptions, plus ou moins reliées les unes aux autres, plus ou moins normatives, qui nous disent, entre autres choses, comment 'fonctionnent' les hommes, à quoi ressemblent notre esprit et celui des autres, comment on doit agir dans des situations précises, quels sont les différents modes de vie possibles et comment il faut s'y conformer » (Bruner, 1991, p. 49).

La culture nous lègue d'ailleurs des « prothèses » (des artefacts, pour nous) qui nous permettent de transcender les limites biologiques « brutes » (les limites de notre mémoire, par exemple). La médiation est envisagée ici comme une interaction amplifiée par un média, un outil qui doit s'envisager suivant deux aspects. D'une 
part, il permet d'effectuer des actions difficilement réalisables sans lui, mais restreint et modèle les actions qu'il médiatise. D'autre part, suivant une perspective vygotskienne, un outil est avant tout, un outil de l'esprit. Cette médiation trouve naturellement ses racines dans les relations humaines et les formes d'une communication rituelle (Bateson, 1971) qui permettent de modéliser les types d'interactions sociales dans des contextes culturels spécifiques. Le second fondement se réfère à Hymes (Hymes, 1982) pour qui, les pratiques langagières quotidiennes des hommes témoignent de la façon dont ceux-ci construisent le social. Dès lors, dégager les règles de fonctionnement de ces pratiques permet de décrire la façon dont les individus associent des modes particuliers de dialogue, d'informations et de transmission de messages avec des lieux et des activités spécifiques. La (re)connaissance est toujours aussi un certain type d'ordre social (Douglas, 1986) dans lequel l'individu devient membre parce qu'on y est prévisible et reconnu. Les usages partagés sont en permanence reconstruits par les faits, les gestes, les échanges (Goffman, 1963). La communauté est alors fondée sur l'acceptabilité et la prévisibilité que doivent offrir en toute situation les membres d'une culture donnée (Goodenough, 1957). Les connaissances communes sont la collection publiquement partagée de principes et de valeurs utilisés à chaque moment pour justifier les conduites (Douglas, 1986) mais aussi, ce tout complexe qui comprend la connaissance, les croyances, l'art, la morale, le droit, les coutumes, et les autres capacités ou habitudes acquises par l'homme en tant que membre de la société.

Enfin, l'innovation trouve sa source dans le dépassement des usages, dans la capacité à produire de nouveaux objets en changeant et en combinant ceux qui existent déjà à partir des connaissances que l'on en a. La principale caractéristique de l'innovation est alors, une capacité à modifier un mode de pensée en fonction du contexte dans lequel elle s'effectue. Le nouvel objet découvre des liens entre des connaissances qui, a priori, n'ont rien en commun et pourtant transformer l'outil de départ.

\section{Anthropologie de la communication}

Le processus artefactuel est l'outil privilégié de l'approche anthropologique de la communication. Il permet de rendre compte de la façon dont les outils et les organisations sociales jouent un rôle dans les processus de médiation instrumentée. L'artefact a une fonction médiatrice sur l'action de l'homme sur le monde : il est utilisé pour agir sur et avec les autres ; il modifie aussi son utilisateur car il sert aussi à agir sur soi-même.

Un artefact est donc toujours créé et utilisé socialement par et pour les buts et les besoins de l'action collective. Cette nature sociale lui confère une fonction de partage des objectifs ; une fonction de coordination de l'activité des membres d'une communauté ; une fonction de communication qui se construit à travers les 
interactions et les médiations sociales. C'est aussi le moyen, donné au membre de la communauté, de se constituer à la fois comme sujet et objet de sa propre activité sur le monde.

Cette approche interactionniste et praxéologique envisage les pratiques comme indexées aux situations dans lesquelles elles sont mises en œuvre. Cette indexicalité positionne la pratique des outils dans un groupe social particulier : c'est « la communication en train de se construire ». Dès lors, le sens donné aux informations n'est plus un " objet » figé relevant d'un système logico-spatio-temporel (i.e. messagerie asynchrone). Il devient l'élément central de la compréhension du contexte et de la pratique dans une situation dynamique de communication collective. En d'autres termes, nous parlons des composants d'un travail collectif avec les outils qui prennent en compte l'importance de la dimension sociale (humaine) dans la création de nouvelles pratiques, sans négliger pour autant le caractère normatif de la technique. Les pratiques sont dès lors, une construction permanente qui n'a rien d'extérieur aux acteurs. Ils ne sont en rien objectifs et l'individu n'est pas un acteur sans histoire, sans passion, il est prisonnier de valeurs qui déterminent son comportement (Garfinkel, 1999). Les pratiques sont toujours en mouvement puisque toujours en construction au travers du « sens commun » (Schütz, 1987) et des savoirs pratiques que les individus sollicitent constamment pour agir sur les " choses » du quotidien. Quotidien qui devient une façon de penser, de questionner, de structurer le monde et apparaît alors, comme une organisation sociale des membres d'une communauté.

Dans cette perspective, notre posture demande d'envisager les situations de communication instrumentée comme un phénomène complexe plus large que les champs disciplinaires qui la questionnent. Cette vision anthropologique de la communication considère les outils comme les constructions d'une culture commune utilisées avec des règles établies dans un contrat (règles implicites de fonctionnement du groupe) situé. Ce sont ces règles implicites qui organisent une communication socialement élaborée et collectivement validée. Notre posture rejette donc les approches disciplinaires qui réduisent les outils à des ressources pour l'action. De fait, nous envisageons les outils comme un système qui met en relation un utilisateur, un outil, une connaissance, une situation et un contexte.

\section{Articulations méthodologiques}

Notre posture méthodologique est donc nécessairement ouverte à un questionnement sur les angles d'observation des outils. En effet, notre démarche ne cherche pas à adopter une méta-méthodologie fondée sur l'anthropologie du système artefactuel qui permettrait de tout observer ou de tout comprendre. Ici, l'option « ce que permettent de faire les outils » vs. "ce qu'on fait avec les outils » permet de dépasser ces craintes. En effet, il conviendrait d'observer ce que le processus artefactuel éclaire aux frontières des champs disciplinaires considérés. Bien sûr, une 
telle approche demande la plus grande prudence dans l'utilisation des théories et des modèles « empruntés ». Nous devons nous attacher à une nécessaire accessibilité (« accountability » Garfinkel, 1999) du discours théorique reconstruit. D’ailleurs ce discours sera confronté aux pratiques situées (« ce qu'ont fait ou dit ») des individus qui les fabriquent au sein de situations contextualisées. Ici, sous la pression de telles contraintes liées à la dynamique de recherche de terrain, l'analyse que nous pourrions conduire prend alors le risque d'une impossible mise à distance du discours scientifique face aux réalités du terrain.

\section{Approche expérimentale}

Le fondement de toute approche expérimentale est de contrôler et faire varier les conditions d'apparition et de déroulement d'un phénomène de façon à déterminer leur part individuelle et collective d'influence sur ce phénomène. Elle demande que le chercheur fasse varier à son gré les conditions de l'expérience. C'est lui qui de façon « objective » va construire ou contrôler les situations qui lui permettent d'observer ces variables. Les situations sont donc toujours construites, artificielles. Dans ce contexte, toute situation observée n'a pour but que de contrôler empiriquement une ou plusieurs hypothèses sur les comportements que l'on a prévu d'observer. Par exemple, si l'on observe les échanges au sein d'un groupe qui réalise une activité, pour que ceux-ci aient du sens pour le dispositif expérimental, le chercheur évacuera les informations non directement concernées par l'activité observée. Ce n'est pas l'individu (homme socialement construit) qui est observé, mais la dimension cognitive du sujet en rapport avec la stratégie qu'il peut mettre en œuvre dans l'activité. La démarche expérimentale consiste à confronter les résultats obtenus avec les prédictions formulées au niveau des hypothèses. Se pose alors le problème de la généralisation des résultats.

Deux points de vue sont donc évacués des approches expérimentales :

- le premier évacue l'aspect dynamique de la relation à autrui, qui ne peut se réduire à une dimension cognitive ;

- le second est l'aspect indexical des variables qui, pour être contextualisée, demande une compréhension située et locale. Il ne peut donc y avoir d'hypothèse de départ (au sens expérimental), les observations ne sont pas confrontées, mais comparées à d'autres. Elles n'ont pas de valeur intrinsèque.

\section{Approche ethnographique}

Dans la logique de ce que nous avons déjà avancé, notre approche est fonctionnaliste (avec pour racines, Malinowski, 1963). Ici, la culture avec toutes ses extensions, (c'est-à-dire le corps complet d'instruments, les privilèges de ses groupes sociaux, les idées, les croyances et les coutumes humaines), constitue un 
vaste dispositif qui met l'homme dans une position optimale pour affronter les problèmes concrets particuliers qui se dressent devant lui dans son adaptation à son environnement. En d'autres termes, cette approche cherche à saisir le point de vue du natif (Bateson, 1971), du membre, son rapport avec la vie, pour se rendre compte de la vision de son monde. Il s'agit moins de décrire les systèmes idéaux de relation que de repérer les modes de comportement et les modes de pensée de personnes rencontrées dans leur situation vécue au quotidien. Cette approche pointe la relativité de l'objectivation des données au point de vue épistémologique de l'observateur. Elle montre qu'une description des conduites humaines crée une interdépendance entre l'observateur et les personnes observées.

Dans notre processus artefactuel, la structuration fonctionnelle des connaissances est celle des modes par lesquels le système cognitif est produit et reproduit dans l'interaction. Ces modes s'ancrent dans les activités des individus compétents, situés dans le temps et dans l'espace et faisant usage des règles et des ressources dans une diversité de contextes d'action. Ainsi, la compétence pourrait être définie comme le savoir maîtrisé, reconnu, dans un domaine d'importance et qui permet de décider dans des cas complexes. Elle autorise la capacité d'anticipation. La compétence des acteurs est aussi le pivot du processus de structuration (Giddens, 1984). Elle est entendue comme tout ce que les acteurs connaissent (ou croient), de façon tacite ou discursive, sur les circonstances de leur action et de celle des autres, et qu'ils utilisent dans la production et la reproduction de l'action. Elle autorise la capacité réflexive. Pour ce qui est de la compétence avec les outils (si on se réfère à la compétence communicationnelle de Hymes, 1974), l'approche fonctionnelle autorise l'individu à s'adapter aux buts qu'il cherche à atteindre. Il utilise les outils pour atteindre ses objectifs, il est capable d'influencer son environnement de manière appropriée et effective. En fait, il obéit à trois traits que l'on trouve dans le système global évoqué plus haut qui recouvre les facultés « supérieures » de prise de décision, d'intentionnalité ou d'organisation : l'individu a conscience de ses outils ; il est motivé pour les utiliser ; il est sensible à la situation dans laquelle ses outils lui permettent d'agir. C'est une adaptation au contexte

Tout l'enjeu de l'approche par le processus artefactuel est donc de clarifier à quel niveau de l'action cognitive et de l'activité en situation interviennent les outils. Comment l'individu s'adapte au contexte pour acquérir de nouvelles connaissances qui le font sauter d'une classe d'objet à une autre ? Comment la médiation s'inscrit dans les outils et les dispositifs?

Dans le cadre de cette approche, deux points sont essentiels aux réponses possibles :

- toute situation est culturellement construite. Dès lors, l'objectif principal est de saisir le point de vue du « natif», du « membre », son rapport avec la vie quotidienne (la situation observée) pour rendre compte de la vision de son monde (son quotidien). Les variables ne sont plus contrôlées ou évoquées, mais données à voir. Il s'agit moins de chercher à généraliser que de décrire des relations à travers des modes de comportement et des modes de pensée dans leurs situations vécues au quotidien ; 
- le second point est la notion de membre, d'appartenance à une communauté de pratique. « $[\ldots]$ L'appartenance sociale à un groupe une condition normalement préalable à toute activité d'analyse et de description des activités sociales de ce groupe. » (Lecerf, 1985, p. 5). En d'autres termes, la notion de membre est perçue ici comme la maîtrise d'un individu, des règles établies dans un contrat situé représentatif des connaissances et pratiques communes en rapport avec l'appartenance à un groupe, à une communauté. "Une fois affiliés, les membres n'ont pas besoin de s'interroger sur ce qu'ils font. Ils connaissent les implicites de leurs conduites et acceptent les routines inscrites dans les pratiques sociales. » (Coulon, 2002, p. 42).

Ces deux points permettent d'envisager la situation d'acquisition de connaissances comme des situations d'interaction localement situées n'appartenant qu'au groupe observé. Les membres du groupe réinventent les modes de communication des savoirs à chaque instant de leur pratique. Si l'on souhaite fournir une description réutilisable (généralisation) de l'observation, le chercheur ne peut plus être extérieur au groupe. Il doit devenir membre de la communauté étudiée, car s'il reste observateur externe, il n'a aucune chance de comprendre ce qu'il voit. Il ne partage pas l'action et ne peut donc ni la comprendre, ni la décrire. Observer une communauté de pratique, c'est être membre. Dès lors, cette posture est fondamentalement interactionniste, seul le chercheur en interaction avec son objet l'autorise à comprendre de l'intérieur les actions des individus qu'il étudie.

\section{Difficultés de mise en auvre}

Le sens que peuvent donner les individus à leur activité n'est jamais indépendant des interactions. Ces interactions se développent suivant une dynamique propre, dans laquelle la notion de groupe correspond à un processus d'action plutôt qu'à une structure.

Toutefois, les ethnométhodes ne nous apportent pas des réponses sur ce qui nous paraît être une véritable méthodologie à approfondir. La difficulté d'une telle méthodologie réside dans la nature des processus à observer : sensations, perceptions, valeurs, connaissances antérieures... Cependant même cette dimension ne suffit pas à caractériser le projet d'observer et d'analyser la dynamique des situations de communication instrumentée. Les questions du rôle de la temporalité sur le but poursuivi en situation demandent de recentrer l'approche sur plusieurs points.

Le premier point qu'il conviendrait d'approfondir réside dans la conception de la situation en tenant compte du caractère situé de l'action. Il nous semble que cette dynamique devrait être plus problématisée. En effet, dire qu'une action située est une action qui s'ajuste à des circonstances locales ou qui prend appui sur des informations indexicales disponibles dans l'environnement ne suffit pas. Particulièrement lorsqu'on pense que les outils considérés comme des artefacts communicationnels ont un rôle essentiel dans la contextualisation de la situation. Il 
semble en effet nécessaire de développer une véritable méthodologie des situations de communication instrumentées. Cette méthodologie pourrait être complétée d'une approche pragmatique de la communication. Cette dernière tiendrait compte du partage des informations structurées en connaissances construites dans le passé. Ces connaissances constituent une sorte d'héritage qui interroge un provisoire décrit, inspiré du courant culturaliste américain qui a introduit l'anthropologie dans l'approche des phénomènes sociaux. Dans ces phénomènes, les formes et les modes de communication seraient les fondements de toute culture. Les organisations (institutions) humaines pourraient se définir comme la somme globale des attitudes, des idées et des comportements partagés par les membres d'un groupe.

Développer une méthodologie des situations de communication instrumentées devrait donc, dans un deuxième temps, éclaircir la nature communicationnelle des outils. En effet, on a trop souvent tendance à amalgamer les outils et les processus dont on s'efforce de rendre compte. Par exemple, parler de relation entre A et B, sous-tend que A et B sont des entités que l'on peut aussi analyser séparément. Cette vision très cartésienne a déjà été remise en question par les approches sur la complexité. Peut-on penser que les relations entre A et B soient limitées par l'échange qui va modifier leurs approches de la situation dans laquelle ils se trouvent? Si la réponse est « oui », alors on envisage implicitement (ou non) que l'interaction soit exclusivement un processus bijectif entre deux systèmes de pensée. Mais alors, comment expliquer que cette interaction soit plus efficace lorsqu'on introduit un outil qu'il soit cognitif ou matériel?

Le processus artefactuel est un processus relationnel, qui construit dans l'esprit de l'individu un système interprétatif de connaissances et des pratiques qui l'aident à comprendre le monde. Un monde qui se construit de manière récursive. Tout artefact donne lieu à une subjectivité. De fait, la pratique ne serait plus l'essence de la relation homme-outil en tant que solution unique mais une solution immédiate, hic et nunc et non projective. Le risque méthodologique d'étudier un « espace-temps » provisoire et dynamique laisse penser à une impossibilité de généralisation d'un instant « $\mathrm{t} \|$ à $《 t+1 »$. Ceci n'est qu'une apparence issue d'une vision trop expérimentale qui limite la lecture de l'objet observé à des aspects linéaires et séquentiels : « on a un état initial et un état final ».

La médiation ne peut donc pas s'inscrire dans une relation individu-outil identique à elle-même. Elle n'apparaît pas à échéances variées, dans un univers qui la reconnaît et qu'elle reconnaît. La médiation est une concordance (accord) transitoire en contexte d'un lien entre les individus. La complexité avec laquelle devrait être envisagé le rôle des outils montre qu'aucune séparation naturelle n'est repérable, qu'aucune frontière supposée ne résiste entre la vision du monde de l'individu et la construction de ses pratiques, au cours de la médiation. Dès lors, les pratiques révèlent de fait, leur existence empirique comme un système de pensée. Celui-ci est significatif de la compréhension des outils et des pratiques qui modifient en temps réel les conditions même de la relation de communication. L'intelligibilité du phénomène appelle donc la mise en œuvre de modes de pensée et de 
constructions formelles qui accompagnent au plus près cette mouvance au sein de laquelle les artefacts nous signifient le monde.

\section{Bibliographie}

Abric J.C., Pratiques sociales et représentations, (s/dir) Paris, PUF, 2édition, 1997.

Agostinelli S., Les Nouveaux Outils de Communication des Savoirs, Paris, l'Harmattan, Communication et Civilisation, 2003.

Agostinelli S., Metge M., Espaces Communs de Communication des Connaissances : E3C. Des espaces partagés de communication au partage des connaissances, Pessac, Presses Universitaires de Bordeaux, Labyrinthes, 2008.

Andreucci C., Ginestié J., « Un premier aperçu sur l'extension du concept d'objet technique chez les collégiens $»$, Didaskalia, $\mathrm{n}^{\circ} 20,2002$, p. 41-65.

Bachelard G., La formation de l'esprit scientifique, Paris, Vrin, 1999.

Bateson G., "Form, Substance, and Difference", G. Bateson (ed.), Steps to an Ecology of Mind, New York, Ballantine Books, 1972, p. 448-466.

Bateson G., La Cérémonie du Naven, Paris, ed. de Minuit, 1971.

Berger P., Luckmann T., La construction sociale de la réalité, Paris, Colin, 1997.

Bertrand C., " Analyse des pratiques professionnelles des enseignants intégrant les outils E », $7^{e}$ Biennale de l'éducation, 2004. http://www.inrp .fr/biennale/7biennale /Contrib / lon gue/122.pdf

Brassac C., «Lev, Ignace, Jerome et les autres... Vers une perspective constructiviste en psychologie interactionniste ", Technologies, Idéologies et Pratiques : revue d'anthropologie des connaissances, vol. XV, $\mathrm{n}^{\circ}$ 1, 2003, p. 195-214.

Bruner J., Car la culture donne forme à l'esprit, Paris, EsHel, 1991.

Callon M., « Eléments pour une sociologie de la traduction », L'année sociologique, Paris, PUF, 1986.

Campillo V., « Analyse et comparaison de pratiques de tutorat pour la formation aux outils », Colloque Euro Méditerranéen et Africain d'Approfondissement sur la FORmation A Distance, CEMAFORAD 4, Université Louis Pasteur de Strasbourg, 9, 10 et 11 avril 2008.

Chaptal A., «Usages prescrits ou annoncés, usages observés. Réflexion sur les usages scolaires du numérique par les enseignants » Document numérique, vol. 10, n 3-4, 2007, p. 81-106.

Coulon A., L'ethnométhodologie, Paris, PUF, coll. Que sais-je ? (5éd.), 2002.

Douglas M., Risk Acceptability According to the Social Sciences, London, Routledge and Kegan Paul, 1986. 
Engeström Y., "Activity theory and individual and social transformation", Y. Engeström, R. Miettinen, Punamäki R.-L. (Eds.), Perspectives on Activity Theory, Cambridge, Cambridge University Press, 1999, p. 19-38.

Engeström Y., "Activity theory as a framework for analysing and redesigning work", Ergonomics, vol. 43, n 7, 2000, p. 960-974.

Folcher V., Rabardel P., « Hommes, artefacts, activités : perspective instrumentale », P. Falzon (ed.), Ergonomie, Paris, PUF, 2004.

Fornel de M., Quéré L., La logique des situations. Nouveaux regards sur l'écologie des activités sociales, Paris, Éditions de l'EHESS, 1999.

Galpérine P.I., " Essai sur la formation par étapes des actions et des concepts ", Talyzina, N. F, De l'enseignement programmé à la programmation des connaissances, Lille, P.U.F., 1980-1966, p. 167-183.

Garfinkel H., Studies in ethnomethodology, Cambridge, Polity Press, 1999-1967.

Gauvain M., "Cultural Tools, Social Interaction and the Development of Thinking". Human Development, vol. 44, n 2-3, 2001, p. 126-143.

Gibson JJ., Shaw R., Bransford J., "The theory of affordances", Perceiving, acting, and knowing. Toward an ecological psychology, New York, Halsted, 1997, p. 67-82.

Giddens A., La constitution de la société : Eléments d'une théorie de la structuration, Paris, PUF, 1984.

Goffman E., Behavior in public places: notes on the social organization of gatherings, New York, Free Press, 1963.

Goffman E., La mise en scène de la vie quotidienne : la présentation de soi, Paris, Minuit, 1973.

Goffman E., Les rites d'interaction, Paris, Minuit, 1974.

Goffman E., The presentation of self in every day life, Paris, Minuit, 1973.

Goodenough W., Cultural Anthropology and Linguistics, Report of the Seventh Annual Round Table Meeting on Linguistics and Language Study, Washington D.C., Georgetown University Press, 1957, p. 167-173.

Hutchins E., "Material anchors for conceptual blends", Journal of Pragmatics, on Conceptual Blending Theory, vol. 37, $\mathrm{n}^{\circ}$ 10, 2005, p. 1555-1577.

Hutchins E., Cognition in the Wild, Cambridge, MIT Press, 1995.

Hymes D., "The anthropology of Communication", Human Communication Theory: Original Essays, New York, Holt, Rinehort and Winston, 1967, p. 1-39.

Hymes D., Foundations in sociolinguistics : an ethnographic approach, University of Pennsylvania, Press Philadelphia, 1974.

Hymes D., Vers la compétence de communication, Paris, Hatier-CREDIF, 1982.

Latour B., « Note sur certains objets chevelus », Nouvelle revue d'ethnopsychiatrie, $\mathrm{n}^{\circ} 27$, 1995, p. 21-36. 
Lave J., Cognition in practice : mind, mathematics, and culture in everyday life, Cambridge, Cambridge University Press, 1988.

Lecerf Y., « Lexique ethnométhodologique », Pratiques de formation, Ethnométhodologies, n $\circ 11-12,1985$.

Léontiev A.N., Activité, conscience, personnalité (1975), Paris, Éditions Sociales, 1984.

Malinowski B., Les Argonautes du Pacifique occidental, Paris, Gallimard, 1963.

Meyerson E., Les fonctions psychologiques et les æuvves, Paris, Albin Michel, 1995.

Moersch C., "Next steps: Using LoTi as a research tool", Learning \& leading with Technology, vol. 29, $\mathrm{n}^{\circ}$ 3, 2001, p. 22-27.

Norman D., « Les artefacts cognitifs », B., Conein, N., Dodier, \& L., Théveneau (eds.), Les objets dans l'action, Raisons Pratiques, vol. 4, 1993, p. 15-35.

Perriault J., La logique de l'usage. Essai sur les machines à communiquer, Paris, Flammarion, 1989.

Rabardel P., Les hommes et les technologies. Approche cognitive des instruments contemporains, Paris, Armand Colin, 1995.

Relieu, M., Salembier, P., Theureau, J., «Activité et Action/Cognition Située », Revue Activites.org, vol. 1, n 2, 2004, p. 1-10.

Robert, R., Une théorie sociétale des outils : penser les outils entre approche critique et modélisation conceptuelle, Paris, Lavoisier, 2009.

Rosch E., "Cognitive representation of semantic categories", Journal of Experimental Psychology, ${ }^{\circ}$ 104, 1975, p. 192-233.

Schütz A., Le chercheur et le quotidien, Paris, Méridiens-Klincksieck, 1987, p. 7-48.

Simondon G., Du mode d'existence des objets techniques, Paris, Aubier, 1989.

Suchman L., Plans and Situated Actions, Cambridge, Cambridge University Press, 1987.

Tikhomirov O., "Man and computer : The impact of computer technology on the development of psychological process", D. Olson, (ed.), Media and symbols: The forms of expression, communication, and education, Chicago, University of Chicago Press, 1987, p. 357-382.

Vygotski L., S., Mind in society. The development of higher psychological process, Cambridge \& London, Harvard University Press, 1978.

Vygotski L., S., Pensée et langage, Paris, Messidor, Editions sociales, 1985.

Weick K. E., The Social Psychology of Organizing, Addison Westley, Reading, MA, 1979.

Weill-Fassina A., Rabardel P., Dubois D., Représentation pour l'action, Toulouse, Octarès, 1993. 